\title{
Myositis Ossificans in a 4-year-old Child
}

\author{
HW Boon, MBBS, Vasanthie B, MS Ortho, Zulkifli 0, MS Ortho \\ Orthopedic Department, Penang Hospital, Georgetown, Malaysia
}

\begin{abstract}
Sprengel's shoulder and myositis ossificans (MO) are rarely seen concomitantly. This report is about a rare case in a 4 year-old girl who presented with right shoulder deformity and pain associated with right proximal arm swelling.
\end{abstract}

Key Words:

Myositis Ossificans, Sprengel's Shoulder

\section{CASE REPORT}

A 4 year old girl with complaints of right shoulder pain for the past 7 months was referred to the orthopaedic clinic. The pain was of gradual onset, slowly increasing in severity over a period of 7 months, but with no preceding history of trauma. The girl was unable to raise her right upper limb fully. Otherwise, she had no other complaints. The patient was born via spontaneous vaginal delivery with an uneventful perinatal history. Her developmental milestones were up to date and she was totally well and normally active prior to the onset of pain. No siblings report similar symptoms.

On general inspection, the girl was well and active. The patient's growth chart was appropriate for age. On examination of the right shoulder, there was mild

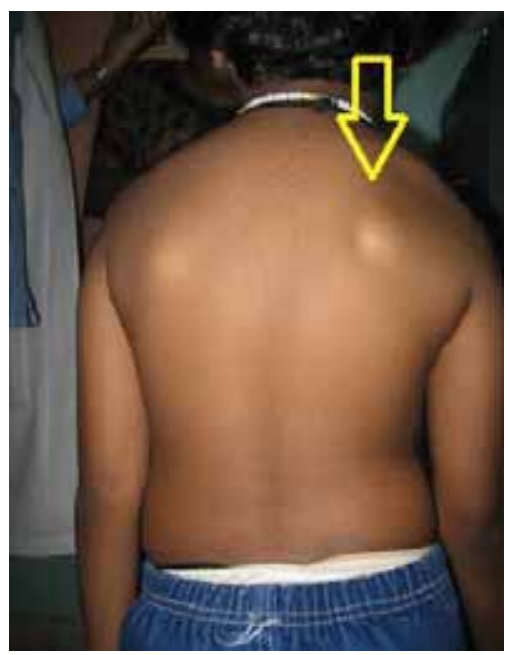

Fig. 1: Shows right Sprengel's shoulder and winging of right scapula. infraspinatus, supraspinatus, and deltoid muscle wasting. The right shoulder was slightly lower than the left shoulder (Fig1), with slight winging of the right scapula. No obvious bony tenderness or swelling was noted. There was a mild restriction of abduction and internal rotation for the right shoulder. Right shoulder power was $5 / 5$. All other limbs appeared normal and no similar problems were noted in any other region. The right shoulder radiograph was normal. Impression at the first consultation was Sprengel's shoulder. The nerve conduction study and electromyogram were normal. MRI of the right brachial plexus was normal as well. During subsequent clinic follow-ups over the next 6 months, the patient's symptoms gradually subsided and range of motion improved.

However, 1 year following the first consultation, she began to experience recurrence of right shoulder pain. At this time, there was a prominent swelling of the right proximal arm measuring $3 \mathrm{~cm} \times 4 \mathrm{~cm}$, which was painful and immobile. MRI of the right shoulder and humerus showed an ill defined lesion measuring $6 \mathrm{~cm} \times 3 \mathrm{~cm} \times 3 \mathrm{~cm}$ in the anterior region of the upper right arm, arising from the deltoid muscle, and suggestive of soft tissue lesion probably due to an inflammatory process or less likely a soft tissue tumour.

On further investigation, the patient's C-reactive protein and ESR were normal. Ultrasound of the right shoulder revealed

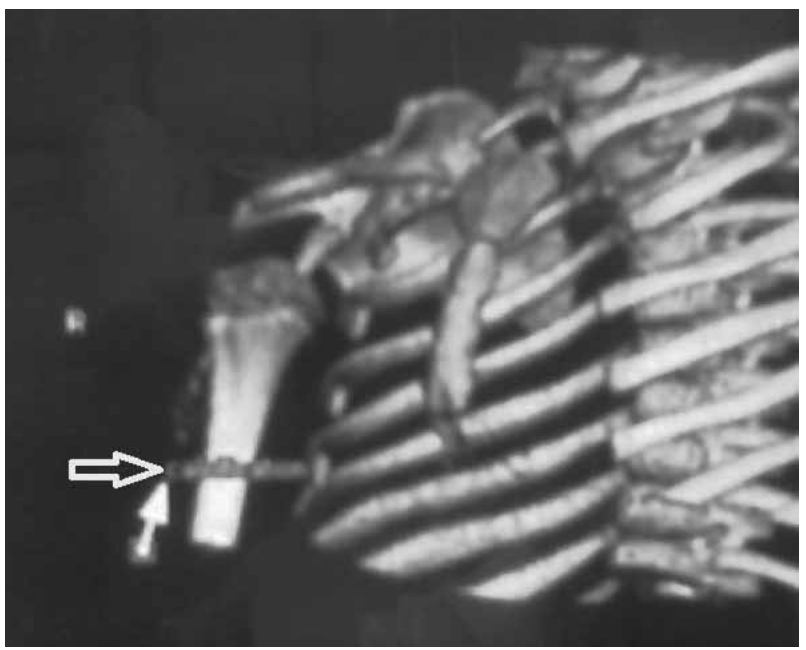

Fig. 2: Shows a fluffy mass of calcifications at right biceps region in $3 \mathrm{D}$ reconstruction $\mathrm{CT}$ scan. 
an ill-defined irregular fluffy mass of calcium in the anterior region of the right deltoid muscle, which was suggestive of myositis ossificans. CT-scan confirmed myositis ossificans of the right bicep muscle.

\section{DISCUSSION}

Myositis ossificans (MO) is an extra-osseous nonneoplastic growth of new bone. The two main recognized MO subtypes are known as traumatic myositis ossificans which occurs following trauma and fibrodysplasia progressive ossificans (FOP) which is a rare, fatal, inherited disorder causing fibrosis and ossification of muscles, tendons and ligaments 1,2,3. Although seemingly similar, these two subtypes have very little in common. Traumatic myositis ossificans is normally seen in the 2nd and 3rd decade and it is commonly seen in the quadriceps and brachialis muscles ${ }^{4}$. The pathogenesis of MO is still remains unclear. In cases of trauma, most believe that ossification occurs following the process of tissue necrosis and hemorrhagic proliferation of fibroblasts and vascular tissue. However non-traumatic MO had been reported in some studies ${ }^{10,11}$. Most believe that repetitive small traumas (not recognized by the patient), infection, inflammation and ischaemia may be the underlying factors that contribute to non-traumatic MO. It is uncommon to see non-traumatic MO, especially in a child. To date, one case has been reported in which a 15 year old adolescent presented with a painful swelling at neck with no history of trauma ${ }^{13}$.

FOP usually begins in the shoulder and spreads progressively from axial to appendicular, cranial to caudal and proximal to distal sites ${ }^{5}$. In most cases, the disease commences during the early years of infancy or childhood and is often discovered by chance. In advanced stages of the disease, extensive ossification in muscles groups involved in joint motion causes mechanical obstruction and eventually permanent deformities.

In contrast, Sprengel's shoulder, or congenital elevation of the scapula, is a result of inadequate caudate movement during development. It is a rare congenital deformity that is commonly associated with most notably Klippel-Feil syndrome as well as congenital scoliosis including cervical scoliosis, fused ribs and spina bifida ${ }^{9}$.

In this case, the patient initially presented with Sprengel's shoulder. Diagnostic testing for other possible conditions associated with Sprengel's shoulder (e.g., brachial plexus pathology) were conducted, but nothing of significance was found. After one year of follow-up, the patient complained of painful swelling at right proximal arm, radiological findings (CT scan and ultrasound) showed a single fluffy mass of calcification appearing only in the right deltoid muscle (fig2). At that time, the working diagnosis was nontraumatic MO. There were no other lesions on the other extremities and the painful MO became less painful and did not progress in size. Currently we are treating the patient conservatively for both MO and Sprengel's shoulder and she is being followed closely for other developments.

This interesting case highlights the concomitant occurrence of two different conditions in the same region of the body. Diagnoses in these cases may be difficult since most clinicians would first attempt to attribute the problems to a single pathology. We found no published reports of an association between the Sprengel's shoulder and MO. 


\section{REFERENCES}

1. Hendifar AE, Johnson D, Arkfeld DG, Jonathan C, Myositis Ossificans. A Case Report. Arthritis Rheum 2005; 53(5): 793-5.

2. Stephen J, Micheal G. Sprengel's deformity. J bone joint Surg 1990; 72(A): 192-7.

3. Nuovo MA, Norman A, Chumas J, Ackerman LV: Myositis ossificans with atypical clinical, radiographic, or pathologic findings: a review of 23 cases. Skeletal Radiol 1992; 21: 87-101.

4. Saussez S, Blaivie C, Lemort M, Chantrain G: Non-traumatic myositis ossificans in the paraspinal muscles. Eur Arch Otorhinolaryngol 2006; 263: 331-5.

5. Kokkosis AA, Balsam A, Lee TK, Schreiber ZJ,: Pediatric nontraumatic myositis ossificans of the neck; Pediatr Radiol 2009; 39(4): 409-12. 